\title{
Study of the production of high-energy neutrinos in the environment of binary-neutron-star mergers
}

\author{
Simone Rossoni, ${ }^{a, *}$ Denise Boncioli ${ }^{b, c}$ and Günter Sigl ${ }^{a}$ \\ ${ }^{a}$ II. Institute for Theoretical Physics, Hamburg University, \\ Luruper Chaussee 149, 22761, Hamburg, Germany \\ ${ }^{b}$ Università dell'Aqula, Dipartimento di Scienze Fisiche e Chimiche, \\ Via Vetoio 40, 67100, L'Aquila, Italy \\ ${ }^{c}$ INFN Laboratori Nazionali del Gran Sasso, \\ Assergi (L’Aquila), Italy \\ E-mail: simone.rossoni@desy.de, denise.boncioli@aquila.infn.it, \\ guenter.sigl@desy.de
}

Neutrinos and gamma-rays are important probes of astrophysical sources and acceleration mechanisms of ultra-high energy cosmic rays (UHECRs). UHECRs can interact with the radiation field and the baryonic material within the source and produce neutrinos in pion decay chains. These neutrinos are subject mostly to redshift and flavour oscillation during their propagation to Earth and contain information on sources otherwise not accessible. We focus on compact objects surrounded by an accretion disk, of the type that are likely end states of a binary-neutron-star (BNS) merger. We model the target photon field in the source as a black body, using a modified version of the code SimProp v2r4 to simulate the propagation and interactions of UHECRs in this environment. We explore various combinations for composition, spectral index, high-energy cutoff of the UHECR primaries. The neutrino fluxes arriving at Earth are compared to the astrophysical IceCube flux, and some constraints on the BNS merger rate can be deduced.

$37^{\text {th }}$ International Cosmic Ray Conference (ICRC 2021)

July 12th - 23rd, 2021

Online - Berlin, Germany

\footnotetext{
*Presenter
} 


\section{Introduction}

Cosmic rays are high-energy ionized nuclei that hit Earth's atmosphere; at the highest energies (i.e. $E \gtrsim 10^{17} \mathrm{eV}$ ) they are called ultra high-energy cosmic rays (UHECRs). They could be produced and accelerated in Galactic and extra-galactic sources, but the acceleration mechanisms and possible classes of sources responsible of such processes are not fully understood.

Since UHECRs are charged particles, they cannot be used as the only astrophysical probes to find out their sources (due to magnetic deflection and interactions) and then to study the physics of high-energy processes. Therefore neutral and stable messengers could be relevant astrophysical probes for ultra-high energy astronomy; in particular, neutrinos travel almost unimpeded through the Universe, thus we could expect them to point directly to their sources.

Extremely energetic environments that might be able to accelerate charged nuclei to the point of producing high-energy neutrinos could be found for instance in supernova remnants, active galactic nuclei and gamma-ray bursts. In the last few years, the IceCube Neutrino Observatory reported the evidence of a diffusive neutrino flux in the $\mathrm{TeV}-\mathrm{PeV}$ energy range consistent with an astrophysical origin [1]. However, the coincidence analysis between neutrino events and the most familiar catalogues of candidate sources did not lead to a definitive answer on the origin of the neutrino flux [2-4]. In this work we considered as alternative neutrino source class the end-state of the binary-neutron-star (BNS) mergers. We assumed the presence of some acceleration mechanism able to accelerate cosmic rays, as suggested in [5], focusing on the study of the interactions of confined UHECRs with the thermal and not-thermal radiation fields originated by the ejected material.

UHECRs confined within their source environment can interact with the ambient photon fields producing charged pions. Therefore neutrinos are produced in the decay chain of charged pions and due to their low cross section they leave the source providing information not accessible through charged particles and photons. The interaction rate $\tau_{i j}^{-1}$ for the process $i$ due to the interaction of a UHECR with the photon field $j$ is given by

$$
\frac{1}{\tau_{i j}}=\frac{c}{2 \Gamma^{2}} \int_{\epsilon_{\mathrm{th}}}^{\infty} d \epsilon \sigma_{i}(\epsilon) \epsilon \int_{\epsilon / 2 \Gamma}^{\infty} d x n_{j}(x) x^{-2}
$$

where $x$ is the photon energy in the source rest frame, $n_{j}(x)$ is the photon spectral energy density (SED, i.e. the number of photons per unit of volume and unit of energy) in the source rest frame, $\sigma_{i}(\epsilon)$ is the total cross section expressed as a function of photon energy in the cosmic-ray rest frame and $\epsilon_{\text {th }}$ is the photon energy threshold in the same frame.

In this work we studied the production of UHECRs and high-energy neutrinos within the environment of a BNS-merger, and the effects of the extra-galactic propagation are also taken into account, after the particles escape from their sources. Indeed, as shown by Greisen, Zatsepin and Kuzmin [6,7], when a charged particle is energetic enough the production of pions can occur with CMB photons during the extra-galactic propagation. This is due to the very efficient relativistic boost of photon energy in the UHECR rest frame. Other possible interactions during propagation are the production of an electron/positron pairs, the photodisintegration of heavy nuclei and the nuclear decay of unstable nuclei. The propagation of UHECRs over extra-galactic distances is also affected by continuous energy loss processes. The fractional energy loss of such adiabatic process is given by $E^{-1} d E / d t=H_{0}$, where $H_{0}$ is the Hubble constant at the present time. 


\section{Computation of UHECRs interactions}

In order to reproduce the propagation of UHECRs over extra-galactic distances many processes need to be taken into account. Continuous energy losses and stochastic processes must be considered to predict the energy spectrum and the mass composition at Earth.

In this work the Monte Carlo code SimProp v2r4 [8] has been used. The energy loss mechanisms previously mentioned are taken into account in SimProp v2r4, as well as the decays of unstable particles. A SimProp run consist of $N$ events injected in the extra-galactic space from a given redshift $z_{\text {inj }}$ with a specific energy $E_{\text {inj }}$ and a mass number $A_{\text {inj }}$ (all these parameters and other options can be specified via command options). After the injection the particle and its interaction products and followed step by step to the Earth at $z=0$. Since the interaction rate reported in Eq.(1) is linear in both $\sigma_{i}(\epsilon)$ and $n_{j}(\epsilon)$, the total interaction rate of stochastic processes can be computed as

$$
\tau_{\mathrm{tot}}^{-1}=\sum_{i, j} \tau_{i j}^{-1}
$$

and the interaction probability of a process is $p_{i j}=\tau_{i j}^{-1} / \tau_{\text {tot }}^{-1}$. The decay of unstable particles is assumed to be instantaneous since the decay lengths are typically much smaller than interaction length scales. While the propagation of electromagnetic particles is not implemented in SimProp $\mathrm{v} 2 \mathrm{r} 4$, neutrinos produced at redshift $z_{v \text {,prod }}$ with energy $E_{\nu \text {,prod }}$ will reach $z=0$ with the energy $E_{\nu, \text { Earth }}=\left(1+z_{\nu, \text { prod }}\right)^{-1} E_{\nu, \text { prod }}$.

In this work the original code SimProp v2r4 has been modified in order to compute the interactions between UHECRs and photons given by a photon SED confined in a source environment. Particles escaping from such interaction region become the injection flux for the extra-galactic propagation that is computed by the original SimProp code.

In order to reproduce the end state of a BNS-merger as reported in [5], the following changes have been implemented in SimProp v2r4. Extra-galactic photon fields (CMB \& EBL) have been replaced by background photon fields within the source environment. We reported these photon SEDs in the left panel of the Figure 1 in which continuous lines correspond to the black body (BB) SEDs, while the dashed lines correspond to the non-thermal (NT) SEDs obtained from [9].

After the merger, the ejected material induces the synthesis of heavy nuclei that produce the high-energy photon emission for days. This leads to the thermal photon field (whose modelling is discussed in the following) powered by the nuclear decay of heavy elements. The thermal emission subsequently decreases until it is dominated by a non-thermal synchrotron emission peaking in the low-energy band. As reported in [9], the non-thermal observation can be modeled by a flux density $\phi(v) \propto v^{-\beta}$, where $v$ is the photon frequency. The parameter $\beta$ is fixed by observations to $\sim 0.6$ and the temporal evolution of $\phi(v)$ can be included in the normalization factor. Therefore we obtained the non-thermal photon SEDs in Figure 1 from the flux density in [9].

Different colors in Figure 1 correspond to different black body temperatures that are related to different times after the merger. The time dependence of the black body temperature has been obtained from the right panel of Figure 2 in [5], considering the so called "Optimistic" case. The latter refers to a source configuration in which the parameters (i.e. $T_{\max } \sim 10^{8} \mathrm{~K}$ ) are chosen to optimize the neutrino flux. The photon energy corresponding to the maximum value of a black body SED can be semi-analytically computed by obtaining the corresponding temperature at a certain 

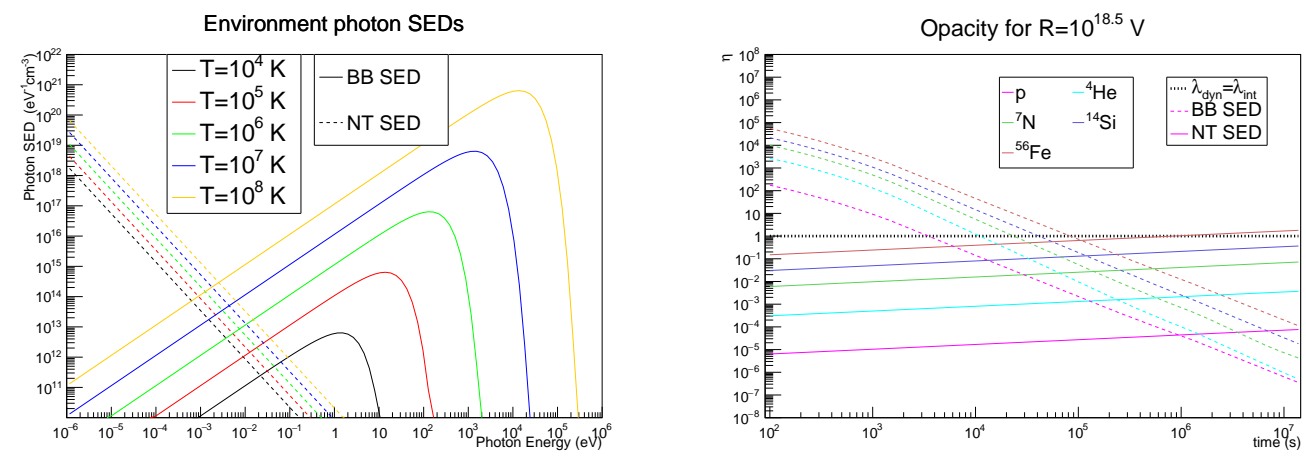

Figure 1: Left: thermal and non-thermal photon SEDs considered in this work at different black body temperatures. Right: source opacities for several nuclei with rigidity $R=10^{18.5} \mathrm{~V}$ obtained with the photon SEDs in the left panel.

time. We then obtained the following parameterisation

$$
\log \left(\frac{t}{1 \mathrm{~s}}\right) \simeq-\frac{1}{2} \log \left(\frac{T}{1 \mathrm{~K}}\right)+6
$$

Therefore, at each temperature the environment can be characterized by a typical radius $\lambda_{\text {esc }}(t)=$ $\beta_{\mathrm{ej}} c t$, where $\beta_{\mathrm{ej}}$ represents the speed of the ejected material in units of speed of light by the merger (hereinafter this parameter is fixed to $\beta_{\mathrm{ej}}=0.3$, as done in [5]). The escape condition is obtained using a simple leaky box model, parametrised by the escape rate $\tau_{\mathrm{esc}}^{-1}=c \lambda_{\mathrm{esc}}(t)^{-1}$, that is compared to the other interaction rates $\tau_{i j}^{-1}$.

In order to evaluate the efficiency of the photo-hadronic interactions in the source environment, we define the total opacity of the source $\eta_{j}$ with respect to a specific photon field $j$ as the ratio between the typical escape length and the total interaction length of a nucleus with the photon field $n_{j}(\epsilon)$ :

$$
\eta_{j}=\frac{\lambda_{\mathrm{esc}}}{\lambda_{j}}=\frac{\sum_{i} \tau_{i j}^{-1}}{\tau_{\mathrm{esc}}^{-1}}
$$

The opacity $\eta_{j}$ is a temperature-dependent quantity and can be interpreted as follows: when $\eta_{j}>1$ the typical interaction length is lower than the dimension of the interaction region and thus the nucleus interacts. On the other hand, for $\eta_{j}<1$ the nucleus can leave the source without interacting. In the right panel of the Figure 1 we report the opacity for several nuclei with rigidity $10^{18.5} \mathrm{~V}$ where the photo-disintegration and photo-pion production have been considered. Interactions of UHECRs with the black body field are very efficient right after the merger, but their contribution decreases rapidly with the cooling down of the source. The non-thermal contribution increases very slowly and it is negligible almost in every time after the merger for the considered nuclei. Similar results were already obtained in [10].

In this work we considered an injection scenario of protons only. Therefore we could consider a source environment where only the thermal black body SED is present for the study of the interactions of UHECRs until $t \simeq 10^{4} \mathrm{~s}$ after the merger (i.e. a black body of $T \simeq 10^{4} \mathrm{~K}$ ). We note that a non-thermal contribution would become relevant in the case of a heavy composition confined within the source environment for $t \gtrsim 10$ days after the merger, which will be studied in the future. 

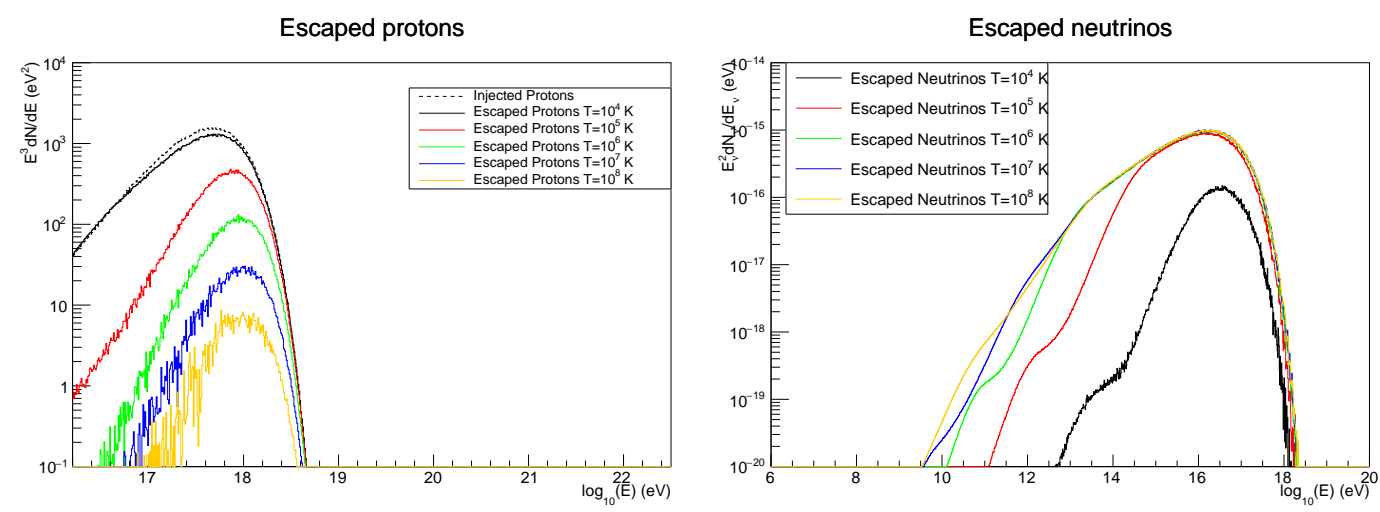

Figure 2: CR (left) and neutrino (right) fluxes escaped from the source environment; CR fluxes are multiplied by $E^{3}$ while the neutrino ones by $E^{2}$. The parameters at injection are $\gamma=1.5$ and $R_{\max }=10^{17.5} \mathrm{~V}$ and the mass composition is pure protons.

\section{Results}

We performed several simulations inside the source environment using the modified version of SimProp v2r4 described in Section 2. Each simulation has been identified by an environment parameter (i.e. the black body temperature) and several UHECRs parameter, such as the injected mass composition, the spectral index at injection and the maximum rigidity.

The energy of injected UHECRs has been extracted from a probability distribution proportional to $\log _{10} E$ in any simulation, defined from $10^{14} \mathrm{eV}$ to $10^{20} \mathrm{eV}$. Different injection configurations have been studied by re-weighing simulation results with an injection spectrum of the form

$$
J_{\text {inj }}(E, Z) \propto E^{-\gamma} \exp \left(-\frac{E / Z}{R_{\max }}\right),
$$

where $\gamma$ is the spectral index and $R_{\max }$ is the high-energy rigidity cutoff. Therefore, by choosing different values of $\gamma$ and $R_{\max }$, the contribution of the low/high-energy part of the injected spectrum can be independently highlighted. Hereinafter the mass composition of the injected spectrum is assumed to be of pure protons.

Regarding the source parameters we fixed five temperature values reported in Figure 2. As described above, at these temperatures the non-thermal contribution is negligible for all nuclei, therefore we only considered the thermal photon field in our simulations. The emission of CRs is larger for low temperatures (black line) than for high temperatures (yellow line) of the black-body, due to the fact that as the temperature decreases, the radius of the source increases slower than the interaction length of the photo-pion production. On the other hand, the production of neutrinos is maximal right after the merger, when the temperature is the highest possible. These considerations are independent of the injection CR parameters, which affect the slope and the maximum energy of the escape spectra only.

Simulations described above have been performed using the resonant photo-pion cross section taken by SOPHIA [11], as implemented in SimProp. Moreover, equation (1) can be analytically approximated assuming a Heaviside function for the photo-pion cross section. Then we computed the interaction rate with the value $\sigma_{p \gamma}=0.5 \mathrm{mb}$ in an energy range from $\epsilon_{\mathrm{th}}$ to $\epsilon_{\mathrm{th}}+\epsilon_{\Delta}$ where 
$\epsilon_{\text {th }}=145 \mathrm{MeV}$ and $\epsilon_{\Delta}=200 \mathrm{MeV}$. However, this approximation leads to an increase in the production of high-energy neutrinos due to the flattening of the photo-pion interaction length for high-energy protons. The result is a large high-energy neutrino flux, not compatible with the experimental limits shown in Figure 3.

In order to predict the diffuse neutrino fluxes, CRs and neutrinos produced within the source environment have been used as inputs for the extra-galactic propagation. Identical sources uniformly distributed are taken into account. Since the in-source simulations do not take into account the redshift of the merger we defined $J_{\text {inj }}^{E G}(E, Z, z=0)$ as the escaped spectra from the source without the redshift dependence. Thereafter we introduced the evolution dependent spectrum for the extragalactic propagation as $J_{\text {inj }}^{E G}(E, Z, z)=J_{\text {inj }}^{E G}(E, Z, z=0)(1+z)^{m}$, where the parameter $m$ defines the cosmological evolution of the sources. Different cosmological evolutions are taken into account for the distribution of sources, such as the case of non-evolution and the one following the starforming rate (SFR). The diffuse fluxes of CRs and neutrinos at Earth were computed using external tools of SimProp v2r4 for the propagation in the extra-galactic space.

We also expect particle injection into the source environment to be described by a time dependent quantity $J_{\mathrm{inj}}(E, Z, t)$. Then the total injected spectrum will be given by the integral over time since the merger. The time evolution of a merger-like event has been taken into account by fixing the initial black body temperature and summing the lower temperature configurations. Therefore we defined two classes of sources: the GW-like class in which the initial temperature is $10^{6} \mathrm{~K}$ and the Optimistic class for $10^{8} \mathrm{~K}$ (as inspired by [5]). Here we reported the results for the GW-like class only because high temperatures of the Optimistic class give rise to neutrino fluxes far above the experimental limits.

We assume in this work that the detected CR flux below the ankle is due to the CRs emitted by BNS mergers. With this assumption, we can derive the normalization of the expected CR flux at Earth at $E<E_{\text {ankle }}$, and as a consequence also the corresponding luminosity density for the source distribution. This fixes also the normalization for the corresponding neutrino fluxes, that include both the neutrinos produced in the source environment and the ones produced in the extra-galactic propagation, that we show in Figure 3 for some choices of the CR spectral parameters at the source and of the cosmological source evolution.

In the upper panels of Figure 3, the different influence of the cosmological evolution on the neutrino flux is visible, being all the other parameters fixed (spectral index $\gamma=1.5$ and maximum energy at acceleration $E_{\max }=10^{18} \mathrm{eV}$ ); while in the left upper plot a distribution of sources with $m=0$ is used, in the right upper plot the case for SFR evolution $(m=3.4)$ is used, and the expected neutrino flux is increased with respect to the non-evolution case. This is visible both for the source neutrinos (blue lines) and for the cosmogenic ones (gray lines). The effect of a higher maximum energy at the source is visible if we compare the left lower plot to the left upper one: the fluxes can reach higher cut-off energy if the maximum energy at the source is higher. In addition, the source neutrinos result in a slightly smaller flux, mainly due to the normalization of the corresponding CR flux. The effect of the spectral index at the source can be inferred by comparing the left upper plot to the right lower one, where a softer spectral index for the injected protons is used, with the final outcome of an increased contribution to the low energy range in the neutrino flux. 

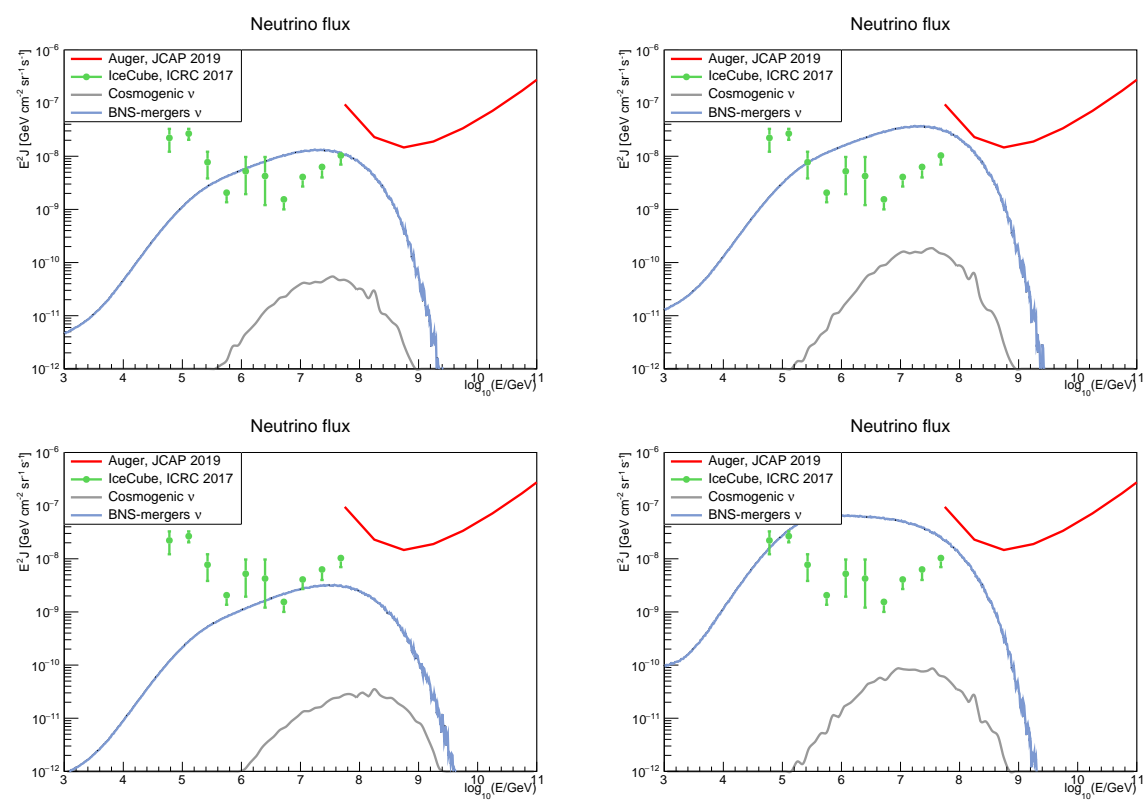

Figure 3: Expected BNS-mergers and cosmogenic neutrino fluxes, with the measured flux by IceCube [12] and the limit for cosmogenic neutrinos by the Pierre Auger Observatory [13]. The expected neutrino fluxes refer to the following parameters of a pure-proton spectrum at injection, for a GW-like source class: $\gamma=1.5$, $R_{\max }=10^{18} \mathrm{~V}$ and $m=0$ (upper left); $\gamma=1.5, R_{\max }=10^{18} \mathrm{~V}$ and $m=3.4$ (upper right); $\gamma=1.5$, $R_{\max }=10^{18.5} \mathrm{~V}$ and $m=0$ (lower left); $\gamma=2.1, R_{\max }=10^{18} \mathrm{~V}$ and $m=0$ (lower right).

\section{Conclusions}

We have reported the high-energy neutrino fluxes from a BNS-merger obtained with a modified version of the code SimProp v2r4. We assumed a pure proton composition and the energy range of the UHECR spectrum injected into the source environment leaving the other parameters free in order to investigate the source phase space of BNS-mergers, that could explain the astrophysical neutrino flux.

A general result is that cosmogenic neutrinos produced during the extra-galactic propagation cannot be responsible of the observed diffuse neutrino flux. For any source configuration we obtained that the high-energy cosmogenic neutrino flux is a subdominant component.

We have shown that the neutrino flux produced in the sources is strongly connected to the time after the merger, being this linked to the temperature of the black-body SED and to the dimension of the source. In this scenario, colder sources seem to be the most favoured. Different spectral parameters also affected the expected flux; in particular we obtained that a hard spectral index and a large rigidity of the protons at the source, could better reproduce the experimental results.

Future outcomes of this work include the computation of the interactions of heavier nuclear species in the source, taking into account both the thermal and the non-thermal SEDs. It will be also relevant to extrapolate the required efficiency for powering the cosmic-ray flux below the ankle, taking into account the luminosity of the SED in a BNS-merger, given the current estimates of the rate of BNS-mergers. 


\section{References}

[1] M. G. Aartsen et al. [IceCube], Science 342 (2013), 1242856

[2] M. G. Aartsen et al. [IceCube], Astrophys. J. 835 (2017) no.1, 45

[3] M. G. Aartsen et al. [IceCube], Astrophys. J. 843 (2017) no.2, 112

[4] K. Bechtol, M. Ahlers, M. Di Mauro, M. Ajello and J. Vandenbroucke, Astrophys. J. 836 (2017) no. 1,47

[5] V. Decoene, C. Guépin, K. Fang, K. Kotera and B. D. Metzger, JCAP 04 (2020), 045

[6] K. Greisen, Phys. Rev. Lett. 16 (1966), 748-750

[7] G. T. Zatsepin and V. A. Kuzmin, JETP Lett. 4 (1966), 78-80

[8] R. Aloisio, D. Boncioli, A. Di Matteo, A. F. Grillo, S. Petrera and F. Salamida, JCAP 11 (2017), 009

[9] R. Margutti, K. D. Alexander, X. Xie, L. Sironi, B. D. Metzger, A. Kathirgamaraju, W. Fong, P. K. Blanchard, E. Berger and A. MacFadyen, et al. Astrophys. J. Lett. 856 (2018) no.1, L18

[10] X. Rodrigues, D. Biehl, D. Boncioli and A. M. Taylor, Astropart. Phys. 106 (2019), 10-17

[11] A. Mucke, R. Engel, J. P. Rachen, R. J. Protheroe and T. Stanev, Comput. Phys. Commun. 124 (2000), 290-314

[12] M. G. Aartsen et al. [IceCube], [arXiv:1710.01191 [astro-ph.HE]].

[13] A. Aab et al. [Pierre Auger], JCAP 10 (2019), 022 